\title{
THE PLANETARY EMISSIVITY LABORATORY (PEL) AT DLR, BERLIN
}

\author{
Alessandro Maturilli, Jörn Helbert, Mario D’Amore \\ Institute for Planetary Research, DLR, Berlin
}

\begin{abstract}
The Institute for Planetary Research has an expertise in spectroscopy of minerals, rocks, meteorites, and organic matter, build up in more than two decades. The available equipment allows spectroscopy from the visible to TIR range using bi-conical reflection, transmission and emission spectroscopy. The institute has an outstanding heritage in designing and building infrared remote-sensing instruments for planetary missions.

The PEL has been operating in various configurations for the last 10 years. The laboratory experimental facilities consist of the main emissivity spectrometer laboratory, a supporting spectrometer laboratory for reflectance and transmission measurements, sample preparation facilities and an extensive collection of rocks and minerals.
\end{abstract}

Index Terms - Spectroscopy, planetary surfaces, emissivity, Mercury, IR

\section{INTRODUCTION}

Remote sensing exploration of planetary surfaces remains the most accurate available method for a global characterization of the crust composition and its implication on planetary evolution scenarios. All the principal planets of the solar system are actually (or have been in the last few years) target of planetary exploration missions, all of them having spacecrafts bringing remote sensing instruments, capable to observe the planets in the (among other spectral regions) very significant visible, near-infrared and thermalinfrared spectral ranges.

The analysis of such measured spectra, especially when regarding the surface composition, takes enormous advantage of previous laboratory activity, very often carried on to support such planetary missions.

For such reasons, in the Planetary Emissivity Laboratory (PEL) at German Aerospace Center (DLR) in Berlin we can, using two distinct instrument working in parallel perform the following kind of measurements on planetary analogue materials or any kind of material of interest:

1) emissivity measurements at high temperatures and under vacuum conditions,

2) bi-directional reflectance measurements at room temperature and under vacuum conditions,
3) Transmission measurements at room temperature and under vacuum conditions,

4) emissivity measurements at low/moderate temperatures and under purging conditions,

5) bi-directional reflectance measurements at room temperature and under purging conditions.

\section{THE PEL SET-UP}

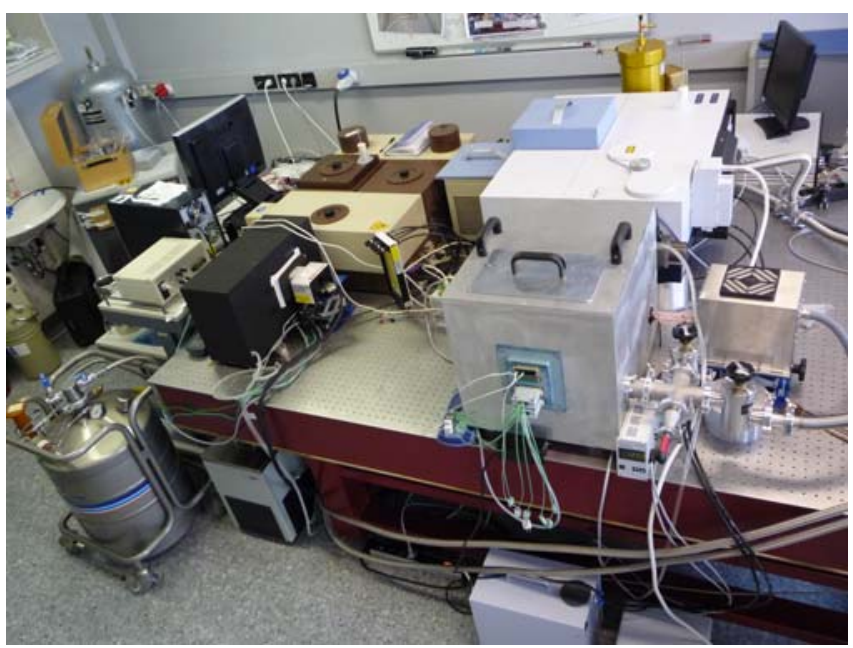

Figure 1. The PEL set-up at DLR in Berlin

The PEL is equipped with a Bruker Vertex $80 \mathrm{~V}$ instrument, coupled to an evacuable high temperatures emissivity chamber and an older Bruker IFS 88 attached to a purged low/moderate temperatures emissivity chamber. The two instruments can work independently in parallel, since they do not share any crucial device. Figure 1 shows the optical table where the two instruments, the external chambers and the other complementary devices are displaced.

The new generation Bruker VERTEX 80V FTIR spectrometer has a very high spectral resolution (better then $0.2 \mathrm{~cm}-1$ ), and a resolving power of better than 300,000:1, and can be operated under vacuum conditions to remove atmospheric features from the spectra. To cover the entire from 1 to $50 \mu \mathrm{m}$ spectral range, two detectors, a liquid nitrogen cooled MTC (1-16 $\mu \mathrm{m})$ and a room temperature DTGS $(15-50 \mu \mathrm{m})$ and two beamsplitter, a $\mathrm{KBr}$ and a Multilayer, are used. However, the DTGS+Multilayer system is usually operated under its full capability, since it allows to measure spectra until $300 \mu \mathrm{m}$. 
The spectrometer is currently coupled to a newly completed planetary simulation chamber (see Figure 2). This chamber can be evacuated so that the full optical path from the sample to the detector is free of any influence by atmospheric gases. The chamber has an automatic sample transport system which allows maintaining the vacuum while changing the samples.

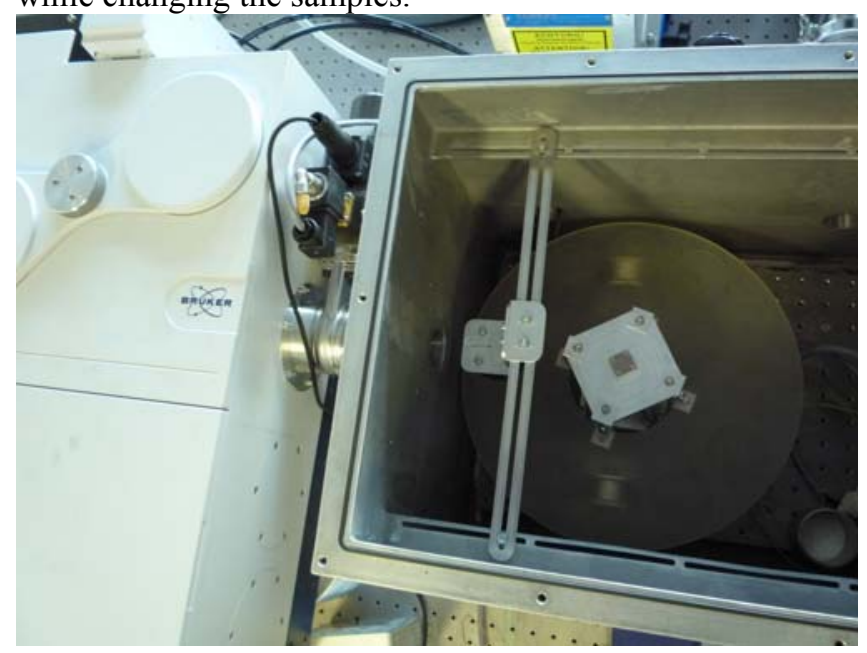

Figure 2. The planetary simulation chamber (top cover was removed)

The induction heating system that is permanently installed in the new chamber allows heating the samples to temperatures of up to $700 \mathrm{~K}$ permitting measurements under realistic conditions for the surface of Mercury. Further details on this device can be found in [1].

The other instrument available in the laboratory is an older Bruker IFS 88 with attached an emissivity chamber, which has been developed at DLR. It is a double-walled water-cooled box with three apertures: a $15 \mathrm{~cm}$ squared door used to insert the cup in the box, a $5 \mathrm{~cm}$ rounded opening through which the beam is directed to the spectrometer and a $5 \mathrm{~cm}$ opening facing the attached blackbody unit. A heater is placed in the chamber and is used to heat the cup with samples from the bottom, from $20^{\circ}$ up to $180^{\circ} \mathrm{C}$. The thermal radiation emitted normal to the surface by the sample or the blackbody is collected by an Au-coated parabolic off-axis mirror and reflected to the entrance port of the spectrometer. The mirror position can be easily set by means of a graduated wheel placed in correspondence of the turning axes of the mirror holder. The chamber (visible in Figure 3, without the cover) is purged with dry air to remove particulates, water vapor and $\mathrm{CO}_{2}$. By means of the cooling mechanism, the chamber temperature can be set and maintained constant. Typical working temperatures are $10^{\circ}$ or even $20^{\circ} \mathrm{C}$, but if needed its temperature can be set to below zero. The chamber and instrument are described in major details in $[2,3]$.

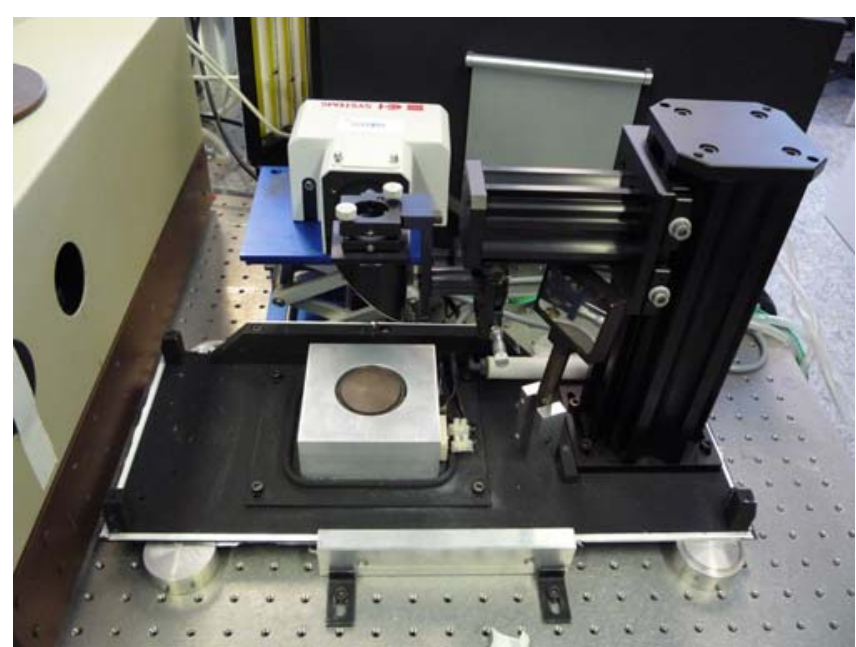

Figure 3. The old emissivity chamber (top cover was removed)

\section{EMISSIVITY MEASUREMENTS}

The VERTEX $80 \mathrm{~V}$ and its attached simulation chamber allows to measure emissivity of minerals at high temperatures between 1 and $50 \mu \mathrm{m}$, under vacuum conditions. Figure 4 shows the spectra of a $25-63 \mu \mathrm{m}$ quartz fraction taken at different temperatures between $180^{\circ}$ and over $600^{\circ} \mathrm{C}$.

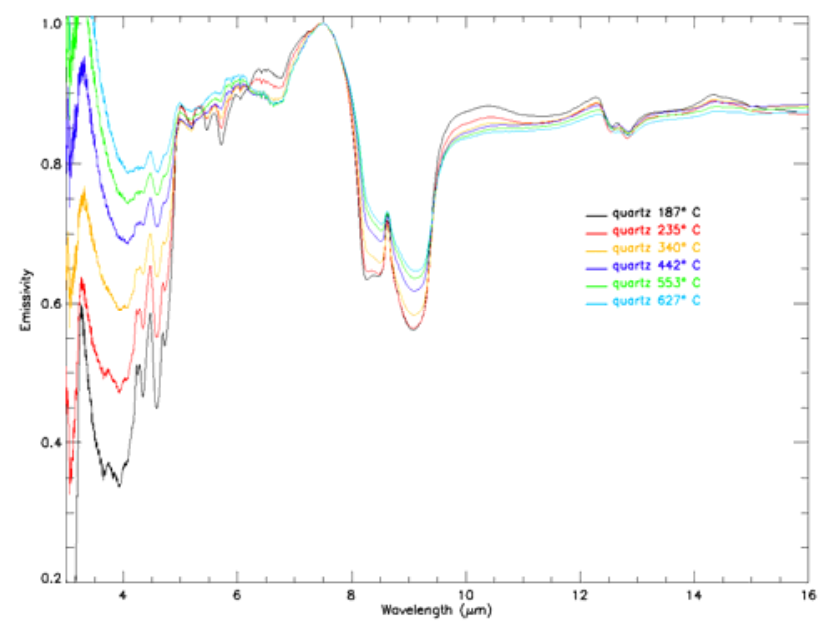

Figure 4. Quartz 25-63 $\mu \mathrm{m}$ at various temperatures.

Similar measurements, in a purged/dry atmosphere and at low/moderate temperatures can be obtained with the Bruker IFS 88 and the coupled emissivity chamber. Figure 5 shows the spectra of quartz in 4 grain size ranges, typically the ones always measured to be ingested in our database, taken with this apparatus. The heater was set to $180^{\circ} \mathrm{C}$ in this experiment. 


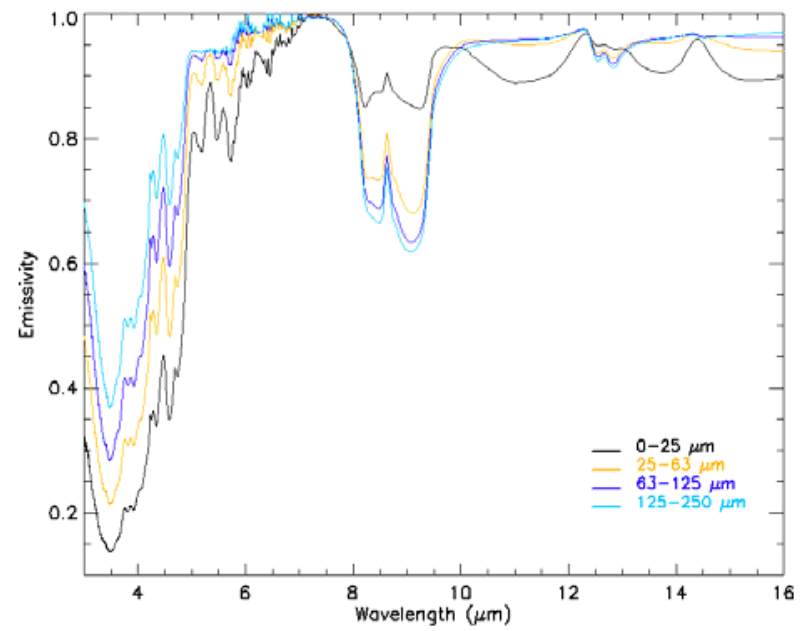

Figure 5.4 quartz separates at $180^{\circ} \mathrm{C}$

\section{REFLECTANCE MEASUREMENTS}

By means of the Bruker A513 accessory (visible in Figure 6) and VERTEX $80 \mathrm{~V}$ instrument, we obtain bidirectional reflectance of minerals, with variable incoming and outcoming angles (between $13^{\circ}$ and $85^{\circ}$ ). We can measure room temperature samples, under purged air or under vacuum conditions, covering the 1 to $50 \mu \mathrm{m}$ spectral range.

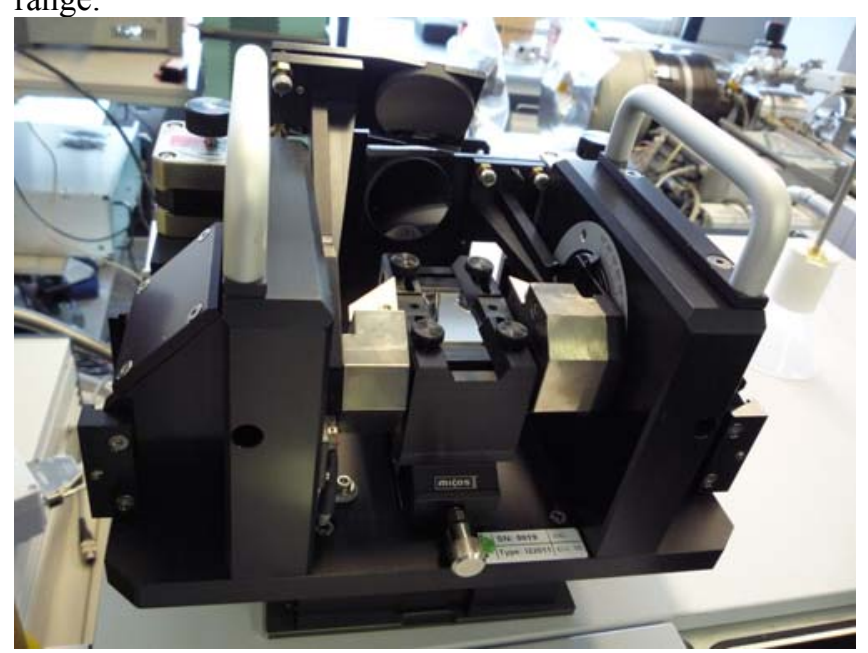

Figure 6. The Bruker A513 reflectance unit

Such measurements can be used even to complete the emissivity measurements we can perform, especially in the case of a very limited amount of the available material. Figure 7 shows the measured reflectance for a sample of quartz in the smaller size separate we usually consider for our materials.

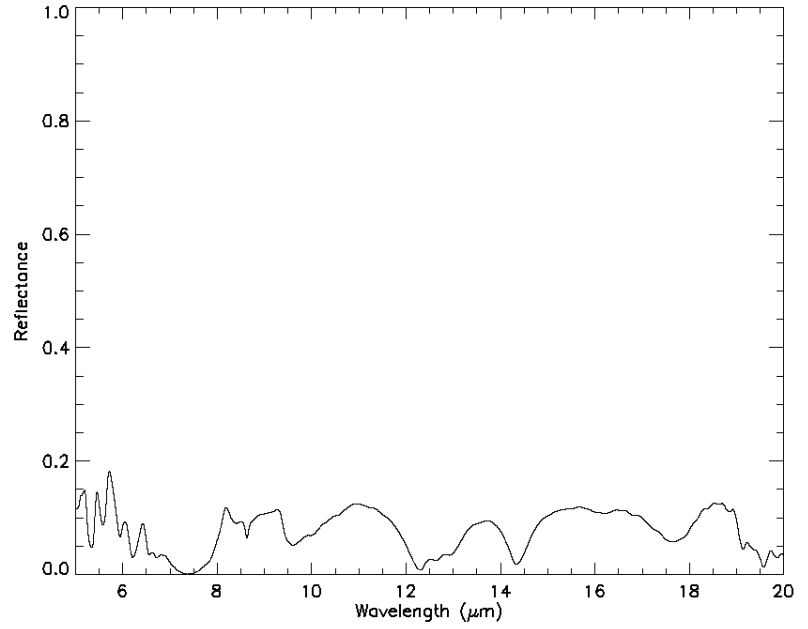

Figure 7. Reflectance spectra of a $0-25 \mu \mathrm{m}$ quartz sample

Similarly, a Harrick Seagull ${ }^{\mathrm{TM}}$ variable angle reflection accessory mounted in the Bruker IFS 88 allows to measure bi-directional reflectance of minerals at room temperature, under purging conditions in the extended spectral range from 0.4 to $16 \mu \mathrm{m}$. Figure 8 shows the device.

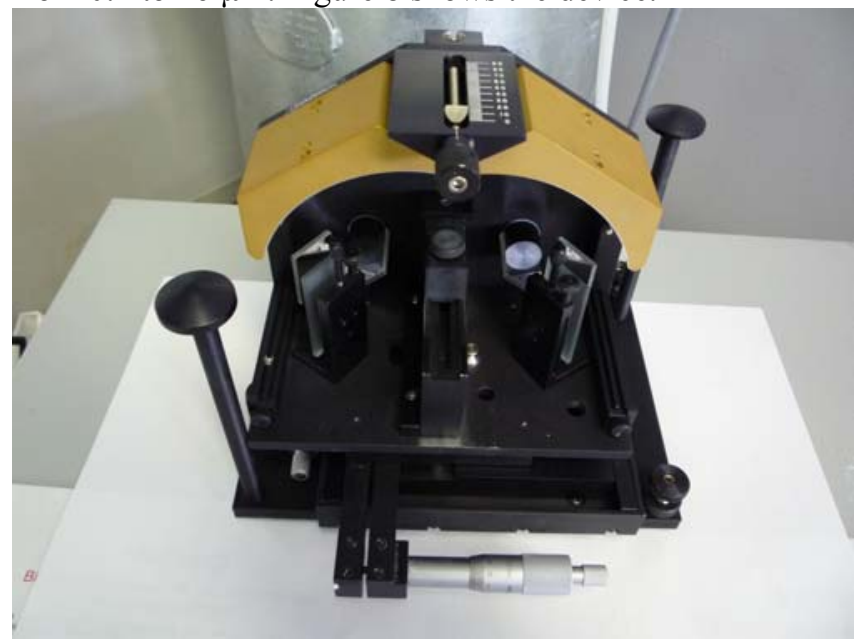

Figure 8. The Harrick Seagull ${ }^{\mathrm{TM}}$ variable angle reflection accessory

\section{TRANSMISSION MEASUREMENTS}

The Bruker A480 parallel beam accessory mounted in the VERTEX $80 \mathrm{~V}$ allows us to accurately measure transmission of thin slabs of material, optical filters, optical window materials, etc, in the complete 1 to $50 \mu \mathrm{m}$ spectral range. Such a device, allows us to avoid refraction (causing focus and lateral beam shifts), typical in this kind of measurements. In Figure 9 the transmission unit is shown. 


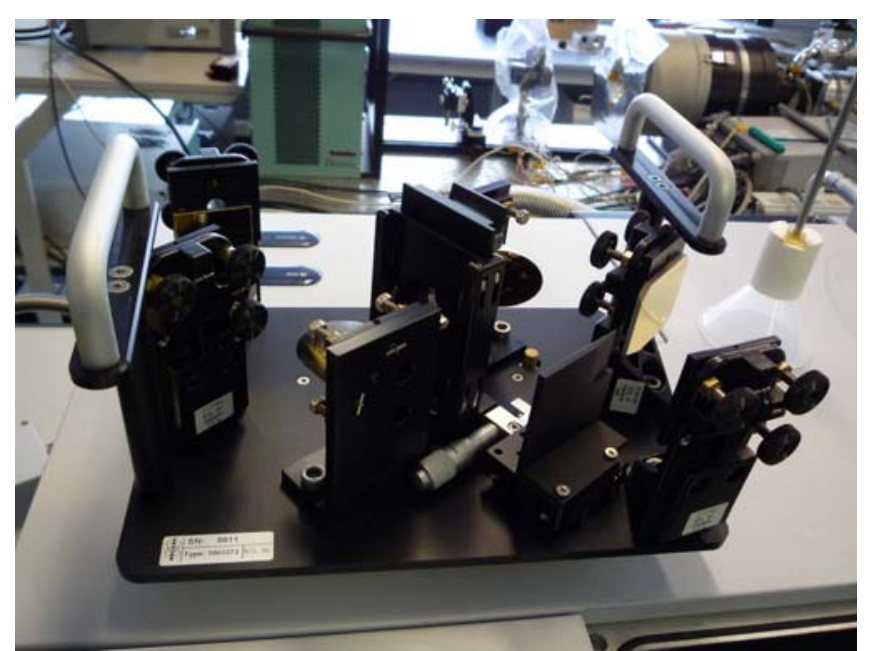

Figure 9. The A480 device for transmission measurements

In Figure 10 the transmission spectral of a filter is shown

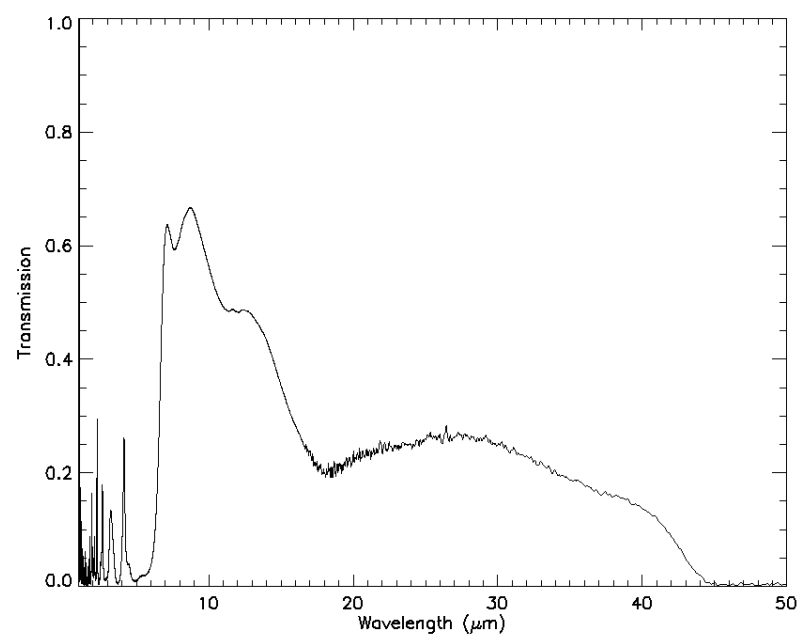

Figure 10. Transmission spectra of a filter

\section{SUMMARY}

The PEL provide the planetary community already today with emissivity measurements highly complementary to existing spectral databases. With the recently completed upgrade the PEL allows unique measurements with a strong focus on airless bodies and extreme conditions as the ones BepiColombo and MESSENGER will encounter at Mercury, over the extremely wide spectral range from 1-50 $\mu \mathrm{m}$ for fine grained samples. Two accessories permit complementary measurements of reflectance and transmission in the same large spectral range, that can be easily extended to until $300 \mu \mathrm{m}$. A second instrument is used to measure emissivity at low/moderate temperatures, and bi-directional reflectance spectra at room temperature of samples, under purged air conditions.

\section{REFERENCES}

[1] J. Helbert, and A. Maturilli, "The emissivity of a fine-grained labradorite sample at typical Mercury dayside temperatures", EPSL 285, Elsevier, pp. 347-354, 2009.

[2] A. Maturilli, J. Helbert, et al., "Emissivity measurements of analogue materials for the interpretation of data from PFS on Mars Express and MERTIS on Bepi-Colombo", PSS 54, Elsevier, pp. 1057-1064, 2006.

[3] A. Maturilli, J. Helbert, and L. Moroz, "The Berlin Emissivity Database (BED)", PSS 56, Elsevier, pp. 420-425, 2008. 